\title{
Time Change of Transfer Function in the Central Japan Anomaly of Conductivity with Special Reference to Earthquake Occurrences
}

\author{
Kazuo Yanagihara and Tetsuro Nagano \\ Kakioka Magnetic Observatory \\ Kakioka, Ibaraki, Japan
}

(Received December 7, 1975)

\begin{abstract}
Electrical conductivity change of the earth interior is confirmed in relation with earthquake occurrences by using transfer functions which connect three components of geomagnetic variation. During 22 months from August 1973 to May 1975, 6 conspicuous changes of transfer functions are found at Kakioka coinciding with 10 large and near earthquakes. High seismic activity extends near the edge of a local conductivity anomaly in the vicinity of Kakioka.
\end{abstract}

1. Time Change of $(\Delta Z / \Delta H)_{\Delta D=0}$ in the Central Japan Anomaly

The characteristics of a conductivity anomaly is not constant for a long time. It changes slowly at least in the central part of Japan (YanAGIHARA, 1972). The change is found in the coefficient $A=(\Delta Z / \Delta H)_{\Delta D=0}$ calculated by the least square method for

$$
\Delta Z=A \cdot \Delta H+B \cdot \Delta D
$$

from three components, $\Delta D, \Delta H$ and $\Delta Z$, of the geomagnetic short period variation observed at Tokyo (1897-1912) and Kakioka (1913-1973) as is shown in Fig. 1. The minimum of $A$ value seems to coincide with the occurrence of a large earthquake $(M=7.8)$ which occurred in 1923 near Tokyo (see Fig. 3). After the minimum the value rapidly increased attaining a maximum in 1940. It is gradually decreasing at present. A cyclic change might be expected. The full range of the change exceeds the error in determining the value.

The annual mean values of the coefficient $A$ for the last 16 years are shown in the lower part of Fig. 1. A kind of cyclic change may be found in the figure, though its range is smaller than that of the slower change. The variation resembles somehow as a whole the trend of the occurrence frequency of earthquakes felt at Kakioka which is shown in the same figure. These may suggest some relation between the conductivity change and earthquake occurrences. Further studies of the relation need a finer time resolution and more accurate determi- 

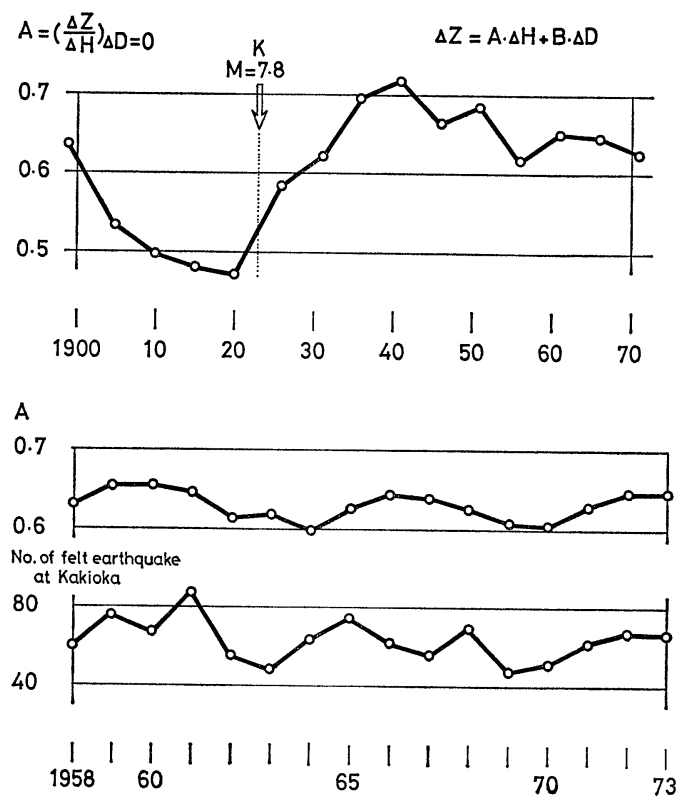

Fig. 1. Time change of $A$-value at Tokyo (1897-1912) and Kakioka (1913-1973).

nation of the value of the coefficient which characterizes the conductivity anomaly.

\section{Time Change of Transfer Function}

A geomagnetic variation includes many frequency (or period) components and the phase of a frequency component is not necessarily the same for every field component. Transfer functions in complex representation must be better than simple coefficients $A$ and $B$ in Eq. (1) to represent a conductivity anomaly. Many studies of the spatial distribution of conductivity anomaly have been carried out by using transfer functions obtained from the spectral analysis of geomagnetic storms or of long continuing disturbances.

However, the accuracy of the value of the transfer function obtained from a single storm is not so high. It is estimated to \pm 0.12 in the $95 \%$ confidence interval for an 80-min component from the analysis of 15 storms (SHIRAKI and YANAGIHARA, 1975). In order to find out any time change which may be smaller than the large spatial change in a conductivity anomaly, it is necessary to use an average of many storms. The use of many storms must induce a rough time resolution.

In this paper isolated disturbances are used to obtain a finer time resolution. 
Let $F_{D}(\omega), F_{H}(\omega)$ and $F_{Z}(\omega)$ be Fourier transforms of $D, H$ and $Z$ components, respectively, of an isolated disturbance whose non-cyclic change is extracted. Transfer functions, $A_{u}(\omega)+i A_{v}(\omega)$ and $B_{u}(\omega)+i B_{v}(\omega)$, connect the Fourier transforms by the linear equation,

$$
F_{Z}(\omega)=\left\{A_{u}(\omega)+i A_{v}(\omega)\right\} F_{H}(\omega)+\left\{B_{u}(\omega)+i B_{v}(\omega)\right\} F_{D}(\omega)
$$

where $\omega=2 \pi / T$ is the angular frequency for the period $T$. From a number of isolated disturbances the value of the transfer function is calculated by the least square method (Everett and Hyndman, 1967). Considering frequent occurrences of geomagnetic disturbance of short duration, this method is better than the use of many storms to get a finer time resolution without the loss of accuracy.

During 22 months from August 1973 to May 1975, transfer functions for Kakioka are calculated for 34 sub-periods. Frequency $\omega$ used in the present analysis is constant at $2 \pi /(80 \mathrm{~min})$, where the corresponding period, $T=80 \mathrm{~min}$, is a predominant one of bay disturbances. Typical values of the calculated transfer functions are $0.65,+0.11,-0.15$ and +0.05 for $A_{u}, A_{v}, B_{u}$ and $B_{v}$, respectively, which are very close to the values, $0.63,+0.11,-0.16$ and +0.06 , respectively, calculated from 15 storms by the power spectral analysis. The signs of $B_{u}$ and $B_{v}$ in the latter values are opposite to those of the original report (ShIRAKI and Yanagihara, 1975), because the positive value of the declination change is taken for eastward in the present analysis instead of westward as in the report. Time changes of the real parts, $A_{u}$ and $B_{u}$, are shown in Fig. 2.

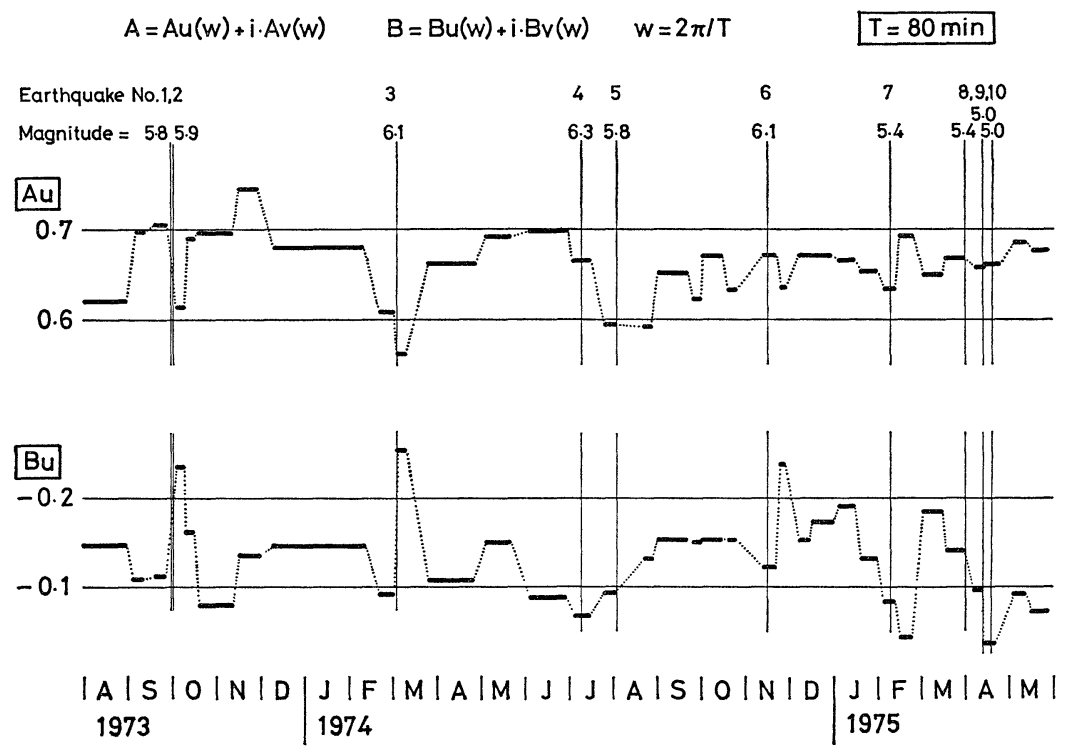

Fig. 2. Time change of transfer function at Kakioka. 
Conspicuous changes of $A_{u}$ and/or $B_{u}$ have occurred five or six times during the period of 22 months. All of the changes are apparently related to earthquake occurrences near Kakioka. It should be noted here that a random choice of an epoch in the present time resolution will meet some earthquake occurrences because 118 earthquakes have been felt at Kakioka during this 22 months. For studies of a relation between the present conductivity change and earthquake occurrences, a reasonable selection of an earthquake is necessary. First, larger earthquakes whose seismic intensity is equal to or larger than III (rather strong) in the Japanese seismic intensity scale is taken into consideration. Out of 118 felt earthquakes, 19 are those. From the 19 earthquakes, those of far epicenter (PS $>50 \mathrm{sec}$ ) or of small amplitude less than $1 \mathrm{~mm}$ are removed. Thus 10 are obtained as large and near earthquakes. They concentrate within $110 \mathrm{~km}$ distance from Kakioka (see Fig. 3) with magnitudes larger than 5. The occurrence times of the 10 earthquakes are marked by the vertical line in Fig. 2. Their magnitudes are also indicated.

The large and near earthquakes (Nos. 1-10) have occurred coinciding with the conspicuous change of the transfer function. A close relationship is understood fairly well. In order to discuss the detailed manner of the change in relation with the earthquake occurrence, the present number of related cases may be too small. The manner may be different for different locations of epicenters.

\section{Discussion in Relation with Tectonic Structure}

The earthquakes felt at Kakioka have occurred most frequently at a depth of several ten kilometers in the vicinity. The region of high seismicity is roughly shown by the shaded area in the map of Fig. 3 for the focal depth of $40-60 \mathrm{~km}$, based upon the seismic activity map compiled by JAPAN METEOROLOGICAL AgENCY (1975). The cross marks of Fig. 3 indicate the epicenters of the 10 earthquakes noted in the preceding section, most of which are located in the shaded area. The remaining two are near the trench in the Pacific Ocean.

The local high values of $A$ or $A_{u}$ are found to the south of Kakioka, superposing upon the general decline of the value towards the inland from the Pacific coast. The small circles of Fig. 3 indicate the sites for which $A$ or $A_{u}$ value is determined neglecting the time change. At the center of the contour lines shown, the value of $A$ exceeds 0.8 , while it is $0.5-0.6$ on the outside where the $A$ value is indicated at each observation site. The high seismicity seems to relate to the local conductivity anomaly. Many earthquakes occur near the inland edge of the anomaly projected from the Pacific side. The directions of the Parkinson vector are nearly southward at most of the sites except a site in the northernmost of the Pacific coast as shown in Fig. 3, where another local anom- 


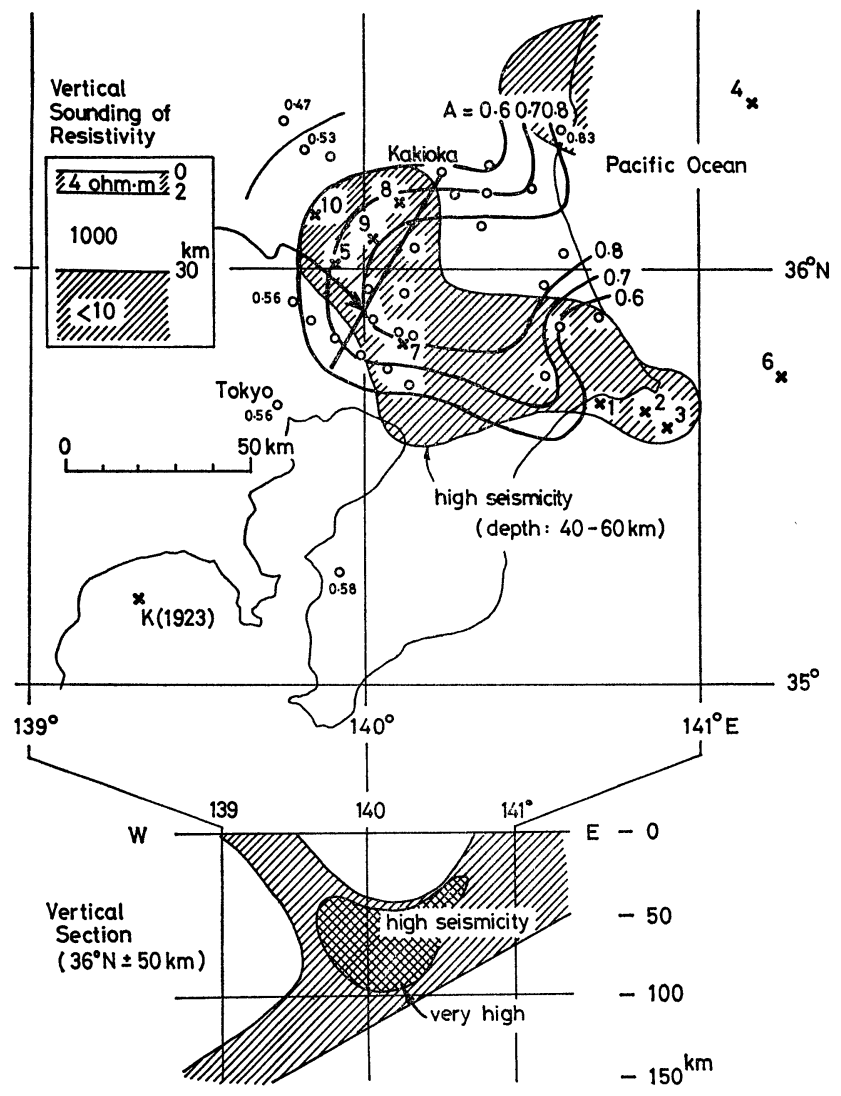

Fig. 3. Distributions of local conductivity anomaly and seismic activity.

aly of conductivity may extend $\left(A_{u}=0.83, B_{u}=-0.35\right.$ by KURUSU, private communication, 1975).

A rough sketch of the vertical distribution of the seismic activity along $36^{\circ} \mathrm{N} \pm 50 \mathrm{~km}$ is shown at the bottom of Fig. 3 based upon TsumurA's (1973) figure. The active region branches between $140^{\circ} \mathrm{E}$ and $141^{\circ} \mathrm{E}$. One branch ascends towards the earth surface from the main zone which gradually descends towards the inland along the plate. At the intersection a very high seismic activity is found.

A possible cause of the local conductivity anomally is a local raise of the conductive layer which is conjectured by Rikitake (1969) to descend towards the inland from the Pacific Ocean in order to interpret the general trend of the central Japan anomaly, and which lies at a deeper level in the vicinity. This is very analogous to the said seismic activity distribution. The assumption of the 
raise of the conductive layer is supported by a resistivity sounding (YANAGIHARA, 1970) which has been made along the line shown in Fig. 3, giving the result of very low resistivity below $30 \mathrm{~km}$. Magnetotelluric sounding also supports the conductive layer (YANAGIHARA and Yokouchi, 1970).

The local raise of the conductive layer may explain the nearly southward direction of the Parkinson Vector at and near Kakioka, which is much different from the general trend of the southeast direction deduced from Rikitake's model, supposing that the northern edge of the conductive layer lies along an east-west line. For the southern edge, a more dense network of observation site will be necessary to discuss the detail.

There seems to be close relations between spatial distributions of seismic activity and conductivity anomaly. The medium of the region may be soft by partial melting or other causes which explain the high conductivity and high seismicity. The time change of the transfer function at Kakioka is found in relation with earthquakes whose epicenters are generally farther than the dimensions of the energy reservoir expected from the magnitudes of the earthquakes. Increase or decrease of conductivity may propagate through the soft medium from the central region of the focus.

Horizontal scale of the local conductivity anomaly is comparable to the depth of the supposed conductive layer which is $30 \mathrm{~km}$ at least. There is still room to suppose that the local anomaly is due to surface currents in the soft sediments. The distribution of the horizontal component of the geomagnetic variation in the region is explained by the effect of a reasonably assumed sediment (YanAGIHARA, 1971). If the spatial distribution of the vertical component is similarly explained by surface currents, the time change of the transfer function may be related with the change of conductivity of the soft sediment. However the obtained distribution of the vertical component is not so easy to explain by a realistic model of the sediment. This suggests an origin in the deeper part of the earth for the local conductivity anomaly and its time change.

\section{REFERENCES}

Everett, J.E. and R.D. Hyndman, Geomagnetic variations and electrical conductivity structure in southwestern Australia, Phys. Earth Planet. Inter., 1, 24-34, 1967.

Rikitake, T., The undulation of an electrically conductive layer beneath the islands of Japan, Tectonophysics, 7, 257-264, 1969.

Japan Meteorological Agency, Seismic activity in and near Japan (1961-1974), Rep. Coord. Comm. Earthq. Predict., 14, 141-147, 1975.

Shiraki, M. and K. Yanagihara, Transfer functions at Kakioka, Memo. Kakioka Mag. Obs., 16, 143-155, 1975.

Tsumura, K., Microearthquake activity in the Kanto district, Publication for the 50th Anniversary of the Great Kanto Earthquake, 1923, pp. 67-87, Earthq. Res. Inst., Tokyo Univ., 1973. 
YANAGIhara, K., Estimate of the deep layer resistivity near Kakioka, Memo. Kakioka Mag. Obs., 12, 115-122, 1970.

Yanagihara, K., Magnetic fields caused by earth surface currents in Kanto district, Memo. Kakioka Mag. Obs., 14, 79-87, 1971.

YANAGIHARA, K., Secular variation of the electrical conductivity anomaly in the central part of Japan, Memo. Kakioka Mag. Obs., 15, 1-11, 1972.

YANAGIHARA, K. and T. Yokouchi, Local anomaly of earth-currents and earth-resistivity, Memo. Kakioka Mag. Obs., 12, 105-113, 1970. 